\title{
SOME PROPERTIES OF TYPE I' STRING THEORY
}

\author{
JOHN H. SCHWARZ \\ California Institute of Technology, Pasadena, CA 91125, USA
}

\begin{abstract}
The T-dual formulation of Type I superstring theory, sometimes called Type I' theory, has a number of interesting features. Here we review some of them including the role of D0-branes and D8-branes in controlling possible gauge symmetry enhancement.
\end{abstract}

\section{Introduction}

I am pleased to contribute to this volume in memory of Yuri Golfand. His name will be remembered by future generations of physicists for his 1971 paper with Likhtmanl which introduced the four-dimensional super-Poincaré algebra for the first time. Recognizing that such a symmetry algebra is a consistent mathematical possibility was certainly a remarkable achievement. It is a curious coincidence that this paper appeared within a few days of Pierre Ramond's paper on fermionic strings 1 Communications were not so good in those days, and the Golfand-Likhtman work was not generally known (at least in the West) for several years. As a result, its influence in driving the development of supersymmetry was not as great as it should have been. In fact, supersymmetric theories in two dimensions were developed to describe the world-sheet theory of RNS strings 3 and this motivated Wess and Zumino to seek fourdimensional analogs 4 Only years later did we understand that RNS strings, properly interpreted, have local 10-dimensional spacetime supersymmetry

The version of the theory that received the most attention prior to 1985 was the one containing both open and closed strings, which Mike Green and I called the type I theory, since it has one ten-dimensional supersymmetry. In 1984 we showed that this theory is inconsistent (due to gauge anomalies) unless the gauge group is chosen to be $\mathrm{SO}(32)$ Then the anomalies cancel, and consistency is achieved. In this manuscript, I propose to review some of the interesting features that appear when one of the spatial dimensions is chosen to be a circle. In this case an alternative $T$ dual description, known as type $\mathrm{I}^{\prime}$, is available. This description gives a different viewpoint for understanding various phenomena, such as gauge symmetry enhancement. The material presented here is not new, though it may be organized somewhat differently than has been done before. 


\section{T Duality}

Let $X^{\mu}(\sigma, \tau)$ denote the embedding functions of a closed string world-sheet in ten-dimensional spacetime. In the case of a trivial flat geometry, the world sheet field equations are simple two-dimensional wave equations. Suppose that one of the nine spatial dimensions, $X^{9}$ say, is circular with radius $R$. Denoting $X^{9}$ by $X$ for simplicity, the general solution of the wave equation is

$$
X=m R \sigma+\frac{n}{R} \tau+\text { periodic terms. }
$$

The parameter $\sigma$ labels points along the string and is chosen to have periodicity $2 \pi$. Thus $m$ is an integer, called the winding number, which is the number of times the string wraps the spatial circle. The parameter $\tau$ is worldsheet time, and correspondingly $p=n / R$ is the momentum along the circle. Single-valuedness of $e^{i p X}$ requires that $n$ is an integer, called the Kaluza-Klein excitation number.

The general solution of the $2 d$ wave equation consists of arbitrary leftmoving and right-moving pieces

$$
X(\sigma, \tau)=X_{L}(\sigma+\tau)+X_{R}(\sigma-\tau) .
$$

In the particular case described above we have

$$
\begin{aligned}
& X_{L}=\frac{1}{2}\left(m R+\frac{n}{R}\right)(\sigma+\tau)+\ldots \\
& X_{R}=\frac{1}{2}\left(m R-\frac{n}{R}\right)(\sigma-\tau)+\ldots
\end{aligned}
$$

$\mathrm{T}$ duality is the world-sheet field transformation $X_{R} \rightarrow-X_{R}, X_{L} \rightarrow X_{L}$ (or vice versa) together with corresponding transformations of world-sheet fermi fields. There are two issues to consider: the transformation of the world-sheet action and the transformation of the space-time geometry. The world-sheet action may or may not be invariant under $\mathrm{T}$ duality, depending on the theory, but the classical description of the spacetime geometry is always radically changed. Let us examine that first:

$$
X=X_{L}+X_{R} \rightarrow X_{L}-X_{R}=\frac{n}{R} \sigma+m R \tau+\ldots
$$

Comparing with eq.(1), we see that this describes a closed string on a circle of radius $1 / R$ with winding number $n$ and Kaluza-Klein excitation number $m$. Thus we learn the rule that under T duality $R \rightarrow 1 / R$ and $m \leftrightarrow n$ In the case of type I or type II superstrings, world-sheet supersymmetry requires that 
$\psi_{R}^{9} \rightarrow-\psi_{R}^{9}$ at the same time. This has the consequence for type II theories of interchanging the IIA theory (for which space-time spinors associated with left-movers and right-movers have opposite chirality) and the IIB theory (for which they have the same chirality). Thus $\mathrm{T}$ duality is not a symmetry in this case - rather it amounts to the equivalence of the IIA theory compactified on a circle with radius $R$ and the IIB theory on a circle with radius $1 / R$. If we compactified on a torus instead, and performed $\mathrm{T}$ duality transformations along two of the cycles, then this would take IIA to IIA or IIB to IIB and would therefore be a symmetry.

In recent years, D-branes have played a central role in our developing understanding of string theory 8 These are dynamical objects, which can be regarded as nonperturbative excitations of the theory. They have the property that open strings can end on them. When they have $p$ spatial dimensions they are called $\mathrm{D} p$-branes. If a $\mathrm{D} p$-brane is a flat hypersurface, the coordinates can be chosen so that it fills the directions $X^{m}, m=0,1, \ldots, p$ and has a specified position in the remaining "transverse" dimensions $X^{i}=d^{i}$ where $i=p+1, \ldots, 9$. An open string ending on such a D-brane is required to satisfy Neumann boundary conditions in tangential directions

$$
\left.\partial_{\sigma} X^{M}\right|_{\sigma=0}=0 \quad m=0,1, \ldots, p,
$$

and Dirichlet boundary conditions in the transverse directions

$$
X^{i}=d^{i} \quad i=p+1, \ldots, 9 .
$$

A remarkable fact, which is easy to verify, is that the T duality transformation $X_{R} \rightarrow-X_{R}$ interchanges Dirichlet and Neumann boundary conditions. This implies that an "unwrapped" D $p$-brane, which is localized on the circle, is mapped by $\mathrm{T}$ duality into a $\mathrm{D}(p+1)$-brane that is wrapped on the dual circle. This rule meshes nicely with the fact that the IIA theory has stable (BPS) $\mathrm{D} p$-branes for even values of $p$ and the IIB theory has stable $\mathrm{D} p$-branes for odd values of $p$. An obvious question that arises is how the wrapped D-brane encodes the position along the circle of the original unwrapped D-brane. The answer is that a type II D-brane has a $\mathrm{U}(1)$ gauge field $A$ in its world volume, and as a result a wrapped D-brane has an associated Wilson line $e^{i \oint A}$. This gives the dual description of position on the circle.

\section{$3 \quad$ Type I Superstrings}

Type IIB superstrings have a world-sheet parity symmetry, denoted $\Omega$. This $Z_{2}$ symmetry amounts to interchanging the left- and right-moving modes on 
the world sheet: $X_{L}^{\mu} \leftrightarrow X_{R}^{\mu}, \psi_{L}^{\mu} \leftrightarrow \psi_{R}^{\mu}$. This is a symmetry of IIB and not of IIA, because only in the IIB case do the left and right-moving fermions carry the same space-time chirality. When one gauges this $Z_{2}$ symmetry, the type I theory results $\theta$ The projection operator $\frac{1}{2}(1+\Omega)$ retains the left-right symmetric parts of physical states, which implies that the resulting type I closed strings are unoriented. In addition, it is necessary to add a twisted sector - the type I open strings. These are strings whose ends are associated to the fixed points of $\sigma \rightarrow 2 \pi-\sigma$, which are at $\sigma=0$ and $\sigma=\pi$. These strings must also respect the $\Omega$ symmetry, so they are also unoriented. The type I theory has half as much supersymmetry as type IIB (16 conserved supercharges instead of 32 - corresponding to a single Majorana-Weyl spinor). This supersymmetry corresponds to the diagonal sum of the $L$ and $R$ supersymmetries of the IIB theory.

This "orientifold" construction of the type I theory has the entire 10d spacetime as a fixed point set, since $\Omega$ does not act on $x^{\mu}$. Correspondingly a spacetime-filling orientifold plane (an O9-plane) results. This orientifold plane turns out to carry -32 units of $R R$ charge, which must be cancelled by adding 32 D9-planes. Rather than proving this, we can make it plausible by recalling that $n$ type I D9-planes carry an $\mathrm{SO}(n)$ gauge group. Moreover, we know that the total charge must be cancelled and that $\mathrm{SO}(32)$ is the only orthogonal group allowed by anomaly cancellation requirements. Correspondingly, these are the unique choices allowed by tadpole cancellation. As a remark on notation, let me point out that instead of speaking of 32 D9-branes, we could equivalently speak of 16 D9-branes and their mirror images. This distinction is simply one of conventions. The important point is that when $n$ type I D9-branes and their $n$ mirror images coincide with an O9-plane, the resulting system has an unbroken $\mathrm{SO}(2 \mathrm{n})$ gauge symmetry.

\section{The Type I' Theory}

We now wish to examine the T-dual description of the type I theory on a spacetime of the form $R^{9} \times S^{1}$, where the circle has radius $R$. We have seen that IIB is T dual to IIA and that type I is an orientifold projection of IIB. Therefore, one should not be surprised to learn that the result is a certain orientifold projection of type IIA compactified on the dual circle $\tilde{S}^{1}$ of radius $R^{\prime}=1 / R$. The resulting $\mathrm{T}$ dual version of type I has been named type IA and Type I' by various authors. We shall adopt the latter usage here.

We saw that $\mathrm{T}$ duality for a type II theory compactified on a circle corresponds to the world-sheet symmetry $X_{R} \rightarrow-X_{R}, \psi_{R} \rightarrow-\psi_{R}$, for the component of $X$ and $\psi$ along the circle. This implies that $X=X_{L}+X_{R} \rightarrow X^{\prime}=$ 
$X_{L}-X_{R}$. In the case of type II theories, we saw that $X^{\prime}$ describes a dual circle $\tilde{S}^{1}$ of radius $R^{\prime}=1 / R$. In the type I theory we gauge world-sheet parity $\Omega$, which corresponds to $X_{L} \leftrightarrow X_{R}$. Evidently, in the T dual formulation this corresponds to $X^{\prime} \rightarrow-X^{\prime}$. Therefore this gauging gives an orbifold projection of the dual circle: $\tilde{S}^{1} / Z_{2}$. More precisely the $Z_{2}$ action is an orientifold projection that combines $X^{\prime} \rightarrow-X^{\prime}$ with $\Omega$. This makes sense because $\Omega$ above is not a symmetry of the IIA theory, since left-moving and right-moving fermions have opposite chirality. However, the simultaneous spatial reflection $X^{\prime} \rightarrow-X^{\prime}$ compensates for this mismatch.

The orbifold $\tilde{S}^{1} / Z_{2}$ describes half of a circle. In other words, it is the interval $0 \leq X^{\prime} \leq \pi R^{\prime}$. The other half of the circle should be regarded as also present, however, as a mirror image that is also $\Omega$ reflected. Altogether the statement of $\mathrm{T}$ duality is the equivalence of the compactified IIB orientifold $\left(R^{9} \times S^{1}\right) / \Omega$ with the type IIA orientifold $\left(R^{9} \times S^{1}\right) / \Omega \cdot \mathcal{I}_{1}$. The symbol $\mathcal{I}_{1}$ represents the reflection $X^{\prime} \rightarrow-X^{\prime}$.

The fixed-point set in the type $\mathrm{I}^{\prime}$ construction consists of a pair of orientifold 8-planes located at $X^{\prime}=0$ and $X^{\prime}=\pi R^{\prime}$. Each of these carries -16 units of $R R$ charge. Consistency of the type $\mathrm{I}^{\prime}$ theory requires adding 32 D8branes. Of these, 16 reside in the interval $0 \leq X^{\prime} \leq \pi R^{\prime}$ and 16 are their mirror images located in the interval $\pi R^{\prime} \leq X^{\prime} \leq 2 \pi R^{\prime}$. Clearly, these D8-branes are the $\mathrm{T}$ duals of the D9-branes of the type I description.

The positions of the D8-branes along the interval are determined in the type I description by Wilson lines in the Cartan subalgebra of $\mathrm{SO}(32)$. Since this group has rank 16, its Cartan subalgebra has 16 generators. Let $A^{I}$ denote the component of the corresponding 16 gauge fields along the circular direction. These correspond to compact U(1)'s, so their values are characterized by angles $\theta_{I}$. These determine the dual positions of the D8-branes to be

$$
X_{I}^{\prime}=\theta_{I} R^{\prime}, \quad I=1,2, \ldots, 16 .
$$

The $\mathrm{SO}(32)$ symmetry group is broken by the Wilson lines to the subgroup that commutes with the Wilson line matrix. In terms of the type $\mathrm{I}^{\prime}$ description this gives the following rules:

- When $n$ D8-branes coincide in the interior of the interval, this corresponds to an unbroken $\mathrm{U}(n)$ gauge group.

- When $n$ D8-branes coincide with an O8-plane they give an unbroken $\mathrm{SO}(2 n)$ gauge group.

In both cases the gauge bosons arise as zero modes of $8-8$ open strings. In the second case the mirror-image D8-branes also contribute. As we will explain 
later, this is not the whole story. Further symmetry enhancement can arise in other ways.

The case of trivial Wilson line (all $A^{I}=0$ ) corresponds to having all 16 D8-branes (and their mirror images) coincide with one of the D8-branes. This gives $\mathrm{SO}(32)$ gauge symmetry, of course. In addition there are two $\mathrm{U}(1)$ factors. The corresponding gauge fields arise as components of the $10 \mathrm{~d}$ metric and B field: $g_{\mu 9}$ and $B_{\mu 9}$. One combination of these belongs to the $9 \mathrm{~d}$ supergravity multiplet, whereas the other combination belongs to a $9 \mathrm{~d}$ vector supermultiplet.

Somewhat more generally, consider the Wilson line

$$
\left(\begin{array}{cc}
I_{16+2 N} & 0 \\
0 & I_{16-2 N}
\end{array}\right)
$$

This corresponds to having $8+N$ D8-branes coincide with the O8-plane at $X^{\prime}=0$ and $8-N$ D8-branes with the O8-plane at $X^{\prime}=\pi R^{\prime}$. Generically this gives rise to the gauge symmetry

$$
S O(16+2 N) \times S O(16-2 N) \times U(1)^{2} .
$$

However, from the S-dual heterotic description of the type I theory, one knows that for a particular value of the radius further symmetry enhancement is

possible. Specifically, for heterotic radius $R_{H}^{2}=N / 8$ one finds the gauge symmetry enhancement

$$
S O(16-2 N) \times U(1) \rightarrow E_{9-N} .
$$

This radius, converted to type I metric, corresponds to $R^{2}=g N / 8$. This symmetry enhancement will be explained from a type $\mathrm{I}^{\prime}$ viewpoint later. There are other interesting extended symmetries such as $\mathrm{SU}(18)$ and $\mathrm{SO}(34)$, which might also be understood from a type I' viewpoint, but will not be considered here.

\section{D0-Branes}

The type I' theory is constructed as a type IIA orientifold. As such, its bulk physics - away from the orientifold planes - is essentially that of the type IIA theory. More precisely, there are number of distinct type IIA vacua distinguished by the difference in the number of D8-branes to the left and the right. When these numbers match, one has the ordinary IIA vacuum. When they don't one has a "massive" IIA vacuum of the kind first considered by Romans 10 In any case, the ordinary IIA vacuum admits various even-dimensional Dbranes. Here I wish to focus on D0-branes. Later we will discuss what happens 
to them when they cross a D8-brane and enter a region with a different IIA vacuum.

D0-branes of the type I' theory correspond to type I D-strings that wrap the compactification circle. The Wilson line on the D-strings controls the positions of the dual D0-branes. A collection of $n$ coincident type $1 \mathrm{D}$-strings has an $\mathrm{O}(n)$ world-volume gauge symmetry. Unlike the case of D9-branes the reflection element is included, so that the group really is $\mathrm{O}(n)$ and not $\mathrm{SO}(n)$. This means that in the case of a single D string it is $O(1)=Z_{2}$. Thus in this case there are two possible values for the Wilson line $( \pm 1)$. The dual type $\mathrm{I}^{\prime}$ description is a single D0-brane stuck to one of the orientifold planes, with the value of the Wilson line controlling which one it is.

A single D0-brane of type I' stuck to an orientifold-plane cannot move off the plane into the bulk. However, a pair of them can do so. To understand this, let us consider a pair of wrapped D strings of type I, coincident in the other dimensions, which carries an $\mathrm{O}(2)$ gauge symmetry. Again, this is $\mathrm{T}$ dual to a pair of type I' D0-branes with positions controlled by the choice of $\mathrm{O}(2)$ Wilson line. The inequivalent choices of Wilson line are classified by conjugacy classes of the $\mathrm{O}(2)$ gauge group. So we should recall what they are. It is important that $\mathrm{O}(2)$, unlike its $\mathrm{SO}(2)$ subgroup, is non-Abelian. Correspondingly, there are conjugacy classes of two types:

- The $\mathrm{SO}(2)$ subgroup has classes labeled by an angle $\theta$. Including the effect of the reflection, inequivalent classes correspond to range $0 \leq \theta \leq$ $\pi$. Such a conjugacy class describes a D0-brane at $X^{\prime}=\theta R^{\prime}$ in the bulk, together with the mirror image at $X^{\prime}=(2 \pi-\theta) R^{\prime}$. We see that to move into the bulk a second (mirror image) D0-brane had to be provided.

- The reflection elements of $\mathrm{O}(2)$ all belong to the same conjugacy class. A representative is the matrix $\left(\begin{array}{cc}1 & 0 \\ 0 & -1\end{array}\right)$. This class corresponds to one stuck D0-brane on each O8-plane.

\section{Brane Creation}

The solutions of massive type IIA supergravity were investigated by Polchinski and Witten 11 who showed that they involve a metric and dilaton that vary in one direction. In the context of the type $\mathrm{I}^{\prime}$ theory this means they vary in all regions for which the number of D8-branes to the left and to the right are unequal. Thus the only case for which this effect does not occur is the $\mathrm{SO}(16) \times \mathrm{SO}(16)$ configuration with D8-branes attached to each of the O8planes (This case is closely related to the M theory description of the $E_{8} \times E_{8}$ theory 12 ) 
We can avoid describing the $X^{\prime}$ dependence of the metric explicitly by using proper distance $s$ as a coordinate along the interval. (This requires holding the other coordinates fixed.) Then one has $0 \leq s \leq \pi R^{\prime}, R^{\prime}=1 / R$. We didn't address the issue earlier, but when we said the interval has length $\pi R^{\prime}$ we really did mean its proper distance. In terms of this coordinate there is a varying dilaton field, and hence a varying string coupling constant $g_{A}(s)$. Only in regions with half of the D8-branes to the left and half to the right is it constant.

The function $g_{A}(s)$ was obtained by Polchinski and Witten by solving the field equations. A more instructive way of obtaining and understanding the result uses the brane creation process. Consider an isolated D0-brane in a region where $g_{A}(s)$ is constant. Now suppose the D0-brane crosses a D8brane to enter a region where $g_{A}(s)$ is varying. What happens is that the D0brane emerges on the other side with a fundamental string stretched between it and the D8-brane. This phenomenon, called Hanany-Witten effect 13 has been derived by a variety of means 14 It occurs in many different settings that are related by various duality transformations. (For example, two suitably oriented M5-branes can cross to give rise to a stretched M2-brane 15 ) The intuitive reason that string creation is required can be understood as follows. The original D0-brane configuration preserved half the supersymmetry and was BPS. Therefore a delicate balance of focus ensured that it was stable at rest. When it crosses the D8-brane (adiabatically) the amount of supersymmetry remains unchanged and so it should still be stable at rest. To be specific, let us consider the D8-brane configuration discussed earlier with $8+N$ D8-branes on the $X^{\prime}=0$ O8-plane and $8-N$ D8-branes on the $X^{\prime}=\pi R^{\prime}$ O8-plane. In this case $N$ fundamental strings should connect the D0-brane to the $X^{\prime}=0$ O8-plane. The BPS condition implies that the mass of the D0-brane should be independent of its position in the interval. Recalling that the mass of a type IIA D0-brane is $1 / g_{A}$, we therefore conclude that for this configuration

$$
M_{D 0}=\frac{1}{g_{A}(0)}=\frac{1}{g_{A}(s)}+N T_{F 1} s
$$

Here $T_{F 1}=\frac{1}{2 \pi}$ is the tension of a fundamental type IIA string (in string units). We therefore see that $g_{A}(s)$ is the reciprocal of a linear function whenever $N \neq 0$. Thus, for $N \neq 0$ it necessarily develops a pole if $R^{\prime}$ is too large.

The mass $M_{D 0}$ can also be computed in the type I picture in terms of a pair of wrapped D strings with Wilson line. The mass is independent of the $\mathrm{O}(2)$ Wilson line, since it is independent of the $X^{\prime}$ coordinate. However, it does depend on the $\mathrm{SO}(32)$ Wilson line. Altogether the mass is a sum of two 
contributions:

$$
M_{D 0}=M_{\text {winding }}+M_{\mathrm{Wilson}} .
$$

The winding term contribution is given by simple classical considerations:

$$
M_{\text {winding }}=2 \cdot 2 \pi R \cdot T_{D 1}=\frac{2 R}{g} .
$$

A more careful analysis is required to obtain the Wilson line contribution

$$
M_{\text {Wilson }}=\frac{N}{4 R} \text {. }
$$

Note that this contribution vanishes for large $R$.

We now come to the main point. There is a special value of $R^{\prime}$, the one for which the coupling diverges at the $X^{\prime}=\pi R^{\prime}$ orientifold plane. In this case

$$
\frac{1}{g_{A}\left(\pi R^{\prime}\right)}=0,
$$

which implies, using eq. (11), that

$$
M_{D 0}=\frac{N}{2 R}=\frac{2 R}{g}+\frac{N}{4 R},
$$

and hence that

$$
R^{2}=g N / 8 .
$$

This is precisely the value that we previously asserted gives the symmetry enhancement $\mathrm{SO}(16-2 N) \times \mathrm{U}(1) \rightarrow E_{9-N}$. The reason that there is symmetry enhancement is that there are additional massless vectors with appropriate quantum numbers. They arise as the ground states of open strings connecting the D8-branes to a stuck D0-brane 16.17 This works because the stuck D0-brane is massless in this case, as a consequence of eq. (15). This accounts for all the extra gauge bosons when $N>2$. In the $E_{7}$ and $E_{8}$ cases, there are additional states attributable to a single bulk D0-brane near $X^{\prime}=\pi R^{\prime}$.

\section{Conclusion}

The study of supersymmetric theories has come a long way since Golfand's pioneering work. I presume that he would be pleased.

\section{Acknowledgments}

I am grateful to O. Bergman for very helpful discussions. This work was supported in part by the U.S. Dept. of Energy under Grant No. DE-FG03-92ER40701. 


\section{References}

1. Yu. A. Golfand and E.P. Likhtman, JETP Lett. 13, 323 (1971).

2. P. Ramond, Phys. Rev. D 3, 2415 (1971).

3. J.-L. Gervais and B. Sakita, Nucl. Phys. B 34, 632 (1971); Y. Aharonov, A. Casher, and L. Susskind, Phys. Rev. D 5, 988 (1972).

4. B. Zumino, p. 367 in Renormalization and Invariance in Quantum Field Theory, ed. E. Caianello (Plenum Press, 1974); J. Wess and B. Zumino, Nucl. Phys. B 70, 39 (1974).

5. F. Gliozzi, J. Scherk, and D. Olive, Nucl. Phys. B 122, 253 (1977); M.B. Green and J.H. Schwarz, Nucl. Phys. B 181, 502 (1981) and Phys. Lett. B 109, 444 (1982).

6. M.B. Green and J.H. Schwarz, Phys. Lett. B 149, 117 (1984).

7. For a review of $\mathrm{T}$ duality see A. Giveon, M. Porrati, and E. Rabinovici, Phys. Rept. 244, 77 (1994), hep-th/9401139.

8. J. Polchinski, Phys. Rev. Lett. 75, 4724 (1995), hep-th/9510017; p. 293 in Fields, Strings, and Duality (TASI 96), eds. C. Efthimiou and B. Greene, World Scientific 1997, hep-th/9611050.

9. A. Sagnotti, p. 521 in Proc. of the 1987 Cargèse Summer Institute, eds. G. Mack et al. (Permagon Press, 1988); N. Ishibashi and T. Onogi, Mod. Phys. Lett. A 4, 161 (1989); G. Pradisi and A. Sagnotti, Phys. Lett. B 216, 59 (1989); P. Hořava, Nucl. Phys. B 327, 461 (1989).

10. L. Romans, Phys. Lett. B 169, 374 (1986).

11. J. Polchinski and E. Witten, Nucl. Phys. B 460, 525 (1996), hepth/9510169.

12. P. Hořava and E. Witten, Nucl. Phys. B 460, 506 (1996), hepth/9510209.

13. A. Hanany and E. Witten, Nucl. Phys. B 492, 152 (1997), hepth/9611230.

14. C.P. Bachas, M.R. Douglas, and M.B. Green, JHEP 9707, 002 (1997), hep-th/9705074; U.H. Danielsson, G. Ferretti, and I.R. Klebanov, Phys. Rev. Lett. 79. 1984 (1997), hep-th/9705084; O. Bergman, M.R. Gaberdiel, and G. Lifschytz, Nucl. Phys. B 509, 194 (1998), hep-th/9705130.

15. S.P. de Alwis, Phys. Lett. B 413, 49 (1997), hep-th/9706142.

16. O. Bergman, M.R. Gaberdiel, and G. Lifschytz, Nucl. Phys. B 524, 524 (1998), hep-th/9711098.

17. C.P. Bachas, M.B. Green, and A. Schwimmer, JHEP 9801, 006 (1998), hep-th/9712086. 